\title{
Prognostic role of PET/CT in endometrial cancer
}

\author{
Ahmet Yanarateş, Emine Budak \\ University of Health Sciences, Izmir Dr. Suat Seren Chest Diseases and Surgery Training and Research Hospital, \\ Department of Nuclear Medicine, Turkey
}

\begin{abstract}
Objectives: The present study evaluates the prognostic value of metabolic parameters related to the primary tumor identified in preoperative fluorodeoxyglucose (FDG) positron emission tomography (PET)/computed tomography (CT) scans in patients with endometrial cancer (EC).

Material and methods: This study included 120 patients with EC who underwent PET/CT in the preoperative period. The patients' age, maximum standardized uptake value (SUVmax), SUVmean, metabolic tumor volume (MTV) and the total lesion glycolysis (TLG) value of the primary tumor on PET/CT; as well as the stage, histological subtype, grade and size of the primary EC; the degree of myometrial invasion (MI) cervical invasion (CI), lymphovascular invasion (LVI), lymph node metastasis (LNM) and distant metastasis (DM) were all recorded. The relationship of these factors with progression-free survival (PFS) and overall survival (OS) was evaluated.

Results: The study included 120 patients with EC with a mean age of $62.3 \pm 0.02$ years. Of the total, 32 patients died around the time of the analysis and 38 patients showed disease progression. The mean OS was $32.7 \pm 1.6$ months and the mean PFS was $30.5 \pm 2.8$ months. No significant relationship was identified between the SUVmax, SUVmean, MTV, TLG values, patient age, tumor size, histology, grade and MI degree, and OS or PFS. Disease stage, LVI, Cl, LNM and DM were identified as prognostic factors for OS and PFS.

Conclusions: The present study found no significant relationship between preoperative PET parameters in EC and OS and PFS, although prospective studies involving a larger number of patients are required.
\end{abstract}

Key words: endometrial cancer; survival; FDG PET/CT; SUVmax; MTV; TLG

Ginekologia Polska 2019; 90, 9: 491-495

\section{INTRODUCTION}

Endometrial cancer (EC) is the most common gynecological malignancy in developed countries [1]. The majority of patients are diagnosed in the early stage [2], and early-stage EC has a good prognosis with 5-year survival reaching $90 \%[3,4]$. That said, the rate of recurrence and the risk of death are high in advanced-stage EC [5] with the 5-year survival in the range of $20-26 \%$ in stage $4 \mathrm{EC}$ [6]. Aside from disease stage, various other prognostic factors have also been described for EC, including histological type, grade, tumor size, myometrial invasion (MI) and lymph node metastasis (LNM) [7]. These prognostic factors can only be determined after extensive surgery [8], and so preoperative prognostic factors that are particulary important for patients who have comorbidities or for young patients who wish to preserve their fertility are sought. Also, some patients may survive even when faced with the same risk factors as those who do not survive, and additive prognostic factors are sought. F-18 fluorodeoxyglu- cose (FDG) positron emission tomography (PET)/computed tomography $(C T)$ is a widely used imaging method in patients diagnosed with malignancy. PET parameters have been suggested to have a prognostic value in various cancer types such as lung cancer [9], esophageal cancer [10] and lymphoma [11-13]. There are, however, only a limited number of studies assessing the value of PET in determining the prognosis of EC.

The present study investigates the relationship between the metabolic parameters of the primary tumor on preoperative PET/CT in patients with EC and overall survival (OS) and progression-free survival (PFS). The study also investigates the prognostic value of certain clinicopathological factors in our patient population.

\section{MATERIAL AND METHODS Patients and follow-up}

This retrospective study included 120 patients with a confirmed histopathological diagnosis of EC between April 
2010 and May 2014 who underwent preoperative F-18 FDG $\mathrm{PET} / \mathrm{CT}$ in our department. None of the patients underwent therapy prior to $\mathrm{PET} / \mathrm{CT}$, and after PET/CT they underwent a total hysterectomy, bilateral salpingo-oophorectomy and pelvic ( \pm paraaortic) lymphadenectomy. Patients received adjuvant therapy involving chemotherapy and/or radiotherapy according to the histological subtype, grade and stages noted by the International Federation of Gynecology and Obstetrics (FIGO) 2009. Progression-free patients were followed up every 3 months for the first two years, every 6 months for 2-5 years and once a year thereafter. As a matter of routine during the follow-up visits, medical histories were obtained, gynecological examinations were made, ultrasonographic data was recorded and serum cancer antigen 125 (CA125) levels were tested. Patients with suspected findings for recurrence underwent a pap smear, CT, MRI and/or PET/CT.

\section{F-18 FDG PET/CT}

Images were taken from the base of the skull to the upper thigh by a PHILIPS GEMINITF 16 Slice PET/CT device. After at least 6 hours of fasting, $0.15 \mathrm{mCi} / \mathrm{kg}$ F-18 FDG was injected into patients with a blood glucose level of less than $200 \mathrm{mg} / \mathrm{dl}$. The patients were allowed to rest in a quiet room for one hour after the injection, after which the images were acquired. PET images were acquired in 9-10 bed positions, for 1.8 min per bed position. The CT images were used for attenuation correction.

The 3D region of interest $(\mathrm{ROI})$ was drawn for the primary tumor, and the maximum standardized uptake value (SUVmax) pertaining to the primary tumor in the region of interest was measured. SUVmean and metabolic tumor volume (MTV) were calculated, considering the recommended [14] 41\% SUVmax as the threshold. The total lesion glycolysis (TLG) was calculated by multiplying the MTV by the SUVmean.

\section{Survival data and statistical analysis}

OS was defined as the time from the date of diagnosis to the date of death from EC, or the time of last observation. PFS was defined as the time from the date of diagnosis to the date of progression or death. Survival curves were created for the patients using the Kaplan-Meier method. SUVmax, SUVmean, MTV, TLG values and patient age were considered as a continuous variable. The relationship between patient age, PET parameters related to the primary tumor (SUVmax, SUVmean, MTV, TLG) and OS and PFS was evaluated using the Cox proportional hazard model. The difference in OS and PFS between the groups classified according to the FIGO stage, the histological subtype, grade, size, MI, cervical invasion $(\mathrm{Cl})$, lymphovascular invasion (LVI) and distant metastasis was evaluated using the log-rank test. A value of $p<0.05$ was considered statistically significant in the analyses.

\section{RESULTS \\ Patient characteristics}

The study included 120 patients with a mean age of $62.3 \pm 0.02$ years (range $42-85$ years) who had been diagnosed histopathologically with EC. According to the FIGO classification, 61 patients had stage 1, 26 had stage 2, five patients had stage 3 and 28 patients had stage 4 disease. Furthermore, 67 patients had an endometrioid histology and the remaining 53 patients had a non-endometrioid histology ( mixed $=27$, undifferentiated $=9$, serous $=7$, mucinous $=5$, squamous $=4$, clear cell =1). Non-endometrioid subtypes were accepted as high-grade carcinoma. Eighteen of the patients with endometrioid carcinoma were grade 1, 35 were grade 2, and 14 were grade 3 . The tumor size was $\geq 4 \mathrm{~cm}$ in 78 patients, $\mathrm{MI}$ was $\geq 50 \%$ in 69 patients, Cl was positive in 29 patients, LVI was positive in 80 patients, pelvic or paraaortic LNM was positive in 13 patients and DM was positive in 17 patients.

\section{Survival}

Of the total sample, 32 died around the time of the analysis and 38 patients showed disease progression. The OS and PFS were $32.7 \pm 1.6$ months and $30.5 \pm 2.8$ months, respectively. When all patients are evaluated together in terms of the PET parameters of the primary tumor, the mean SUVmax was $17.3 \pm 0.07$, the mean SUVmean was $8.6 \pm 0.2$, the mean MTV was $30.8 \pm 0.01 \mathrm{~mL}$ and the mean TLG was $295.2 \pm 0.002 \mathrm{~g}$. No significant relationship was identified between SUVmax, SUVmean, MTV, TLG values, patient age and OS and PFS ( $p>0.05$ ) (Tab. 1). Figures 1 and 2 present the PET parameters and survival data of the sample cases. There were no significant differences in terms of OS and PFS between the patients grouped according to tumor size $(<4 \mathrm{~cm}$ vs $\geq 4 \mathrm{~cm}$ ), histology (endometrioid vs non-endometrioid), grade (grade $1-2$ vs grade 3 ) and $\mathrm{MI}$ degree ( $<50 \%$ vs $\geq 50 \%$ ) $(p>0.05)$. OS and PFS were lower in patients with advanced stage than in patients with early stage, in LVI (+) patients than in $\mathrm{LVI}(-)$ patients, in $\mathrm{Cl}(+)$ patients than in $\mathrm{Cl}(-)$ patients, in LNM (+) patients than in LNM (-) patients, and in DM (+) patients than in DM (-) patients (Tab. 2).

\begin{tabular}{|l|l|l|l|l|}
\hline \multicolumn{5}{|c|}{ Table 1. Survival analysis results of PET parameters and age } \\
\hline & \multicolumn{2}{|l|}{ Overall survival } & \multicolumn{2}{l|}{ Progression free survival } \\
\cline { 2 - 5 } & p & HR & p & HR \\
\hline SUVmax & 0.800 & 1.018 & 0.544 & 1.045 \\
\hline SUVmean & 0.916 & 0.978 & 0.667 & 0.914 \\
\hline MTV & 0.865 & 0.997 & 0.098 & 1.016 \\
\hline TLG & 0.615 & 1.001 & 0.644 & 0.999 \\
\hline Age & 0.820 & 1.007 & 0.414 & 1.022 \\
\hline
\end{tabular}

SUVmax - maximum standardized uptake value; MTV — metabolic tumor volume; TLG — total lesion glycolysis 
A

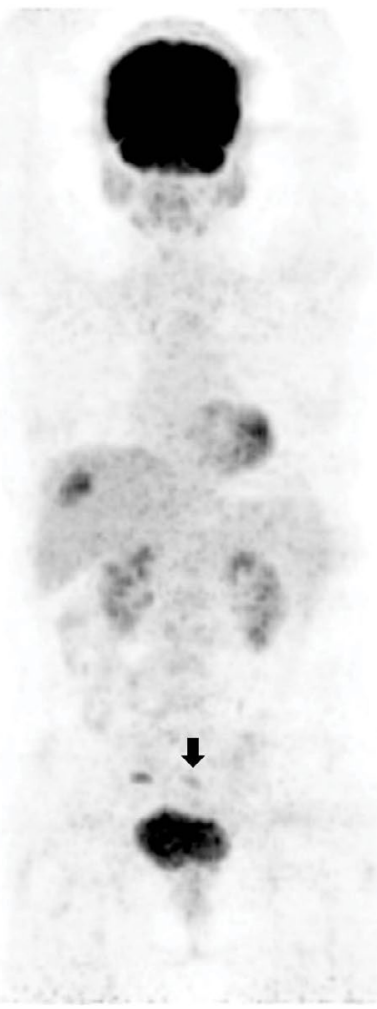

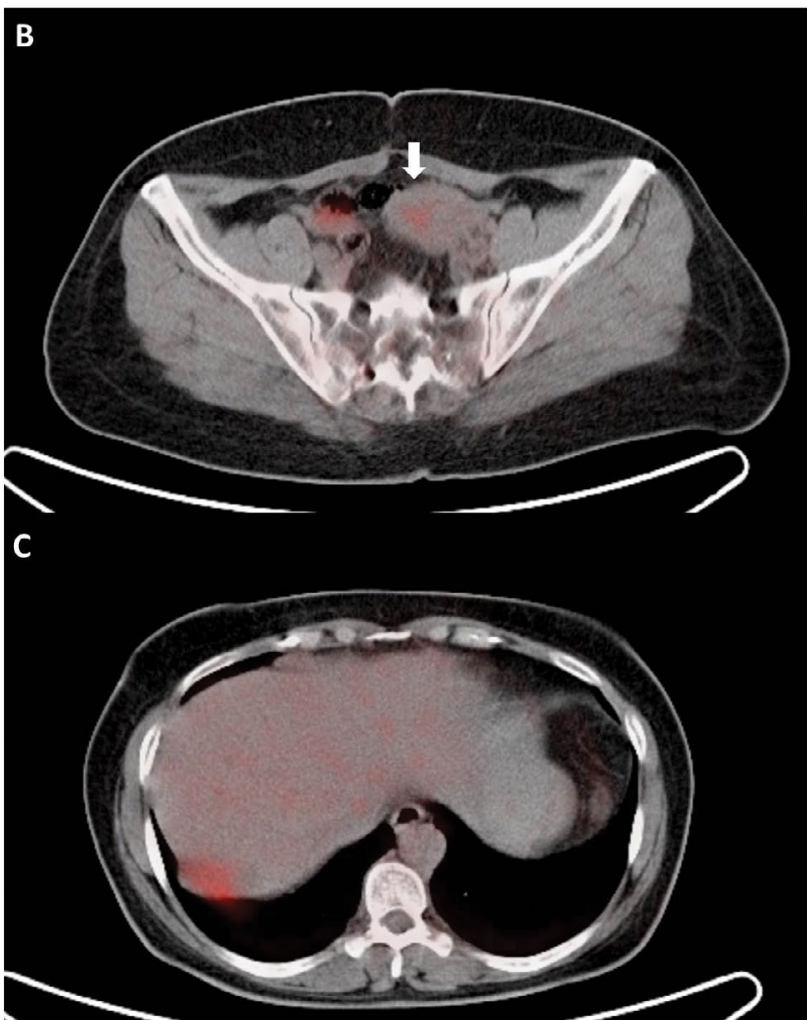

Figure 1. The MIP $(A)$ and selected axial PET/CT $(B, C)$ images of a 56-year-old patient with stage $4 B$ undifferentiated endometrial cancer and a capsular implant in the liver are presented. Among the PET parameters of the primary tumor of the patient (A,B; arrows), SUVmax, SUVmean, MTV and TLG were found to be $3.8,3,1.2 \mathrm{~mL}$ and $3.6 \mathrm{~g}$, respectively. The progression-free survival and overall survival of this patient were 6 months and 17 months, respectively

A

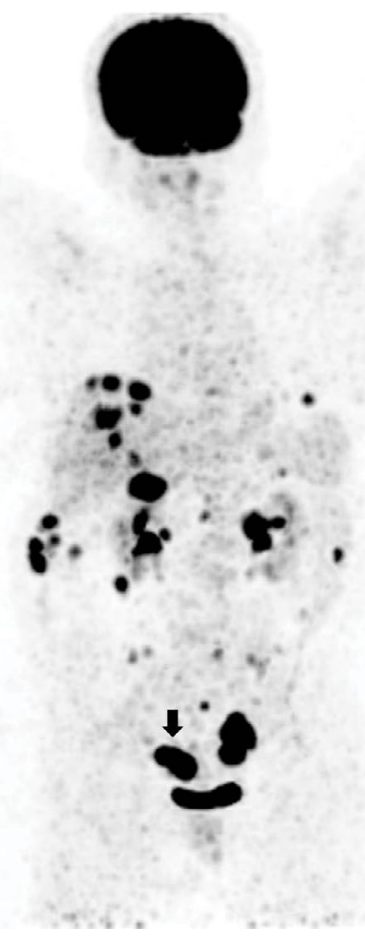

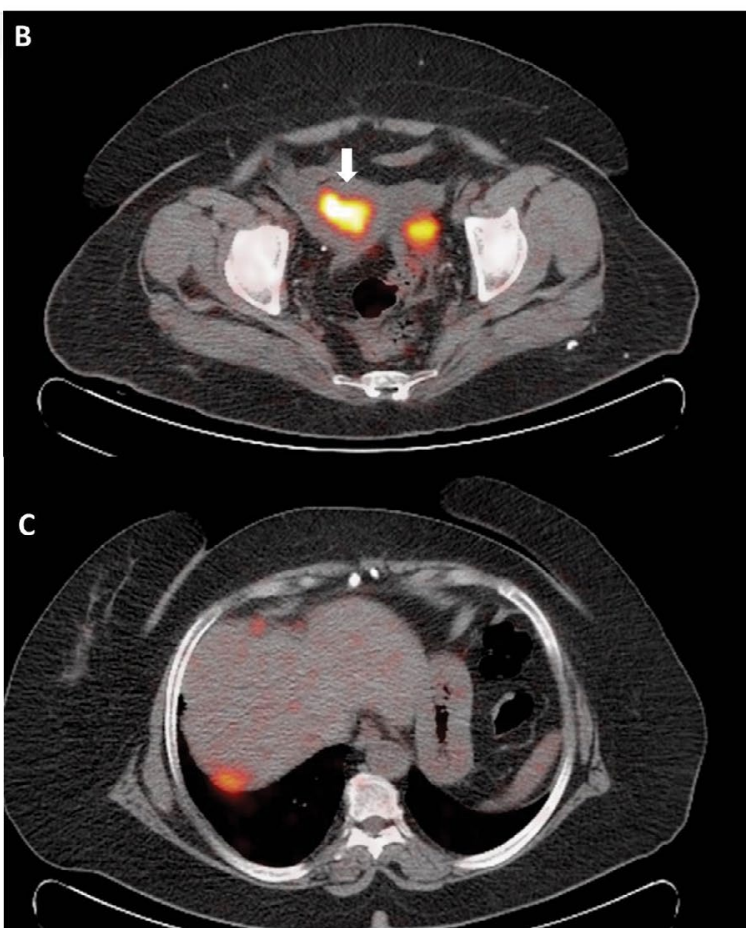

Figure 2. The MIP $(A)$ and fusion $(B, C)$ images of a 61 -year-old patient with stage $4 B$ mix endometrial cancer and a capsular implant in the liver are presented. The PET parameters of the primary tumor of the patient (A, B; arrows) were calculated as follows: SUVmax: 24.4, SUVmean: 8.5, MTV: 17.8 $\mathrm{mL}$, and TLG: $151.3 \mathrm{~g}$. The progression-free survival and overall survival of this patient were 8 months and 16 months, respectively 
Table 2. Comparison of overall survival and progression free survival between the groups

\begin{tabular}{|c|c|c|c|c|c|}
\hline & $\mathbf{n}$ & $\begin{array}{l}\text { OS } \\
\text { (mean } \pm \mathrm{SE} \text {, months) }\end{array}$ & $\mathbf{p}$ & $\begin{array}{l}\text { PFS } \\
\text { (mean } \pm S E \text {, months) }\end{array}$ & $\mathbf{p}$ \\
\hline Stage 1-2 & 87 & $43.7 \pm 1.3$ & \multirow{2}{*}{$<0.001^{*}$} & $42.7 \pm 1.5$ & \multirow{2}{*}{$<0.001^{*}$} \\
\hline Stage 3-4 & 33 & $21.9 \pm 4.5$ & & $13.9 \pm 4.1$ & \\
\hline Tumor size $<4 \mathrm{~cm}$ & 42 & $38.3 \pm 3.0$ & \multirow{2}{*}{0.881} & $36.6 \pm 3.8$ & \multirow{2}{*}{0.733} \\
\hline Tumor size $\geq 4 \mathrm{~cm}$ & 78 & $38.8 \pm 2.2$ & & $36.0 \pm 2.5$ & \\
\hline LVI (-) & 40 & $44.3 \pm 2.0$ & \multirow{2}{*}{$0.027^{*}$} & $44.3 \pm 2.0$ & \multirow{2}{*}{$0.007^{*}$} \\
\hline LVI (+) & 80 & $36.0 \pm 2.6$ & & $31.9 \pm 3.0$ & \\
\hline Endometroid & 67 & $42.1 \pm 2.1$ & \multirow{2}{*}{0.156} & $41.1 \pm 2.3$ & \multirow{2}{*}{0.083} \\
\hline Nonendometroid & 53 & $36.3 \pm 2.9$ & & $32.1 \pm 3.4$ & \\
\hline Grade1-2 & 53 & $42.8 \pm 2.1$ & \multirow{2}{*}{0.190} & $41.6 \pm 2.3$ & \multirow{2}{*}{0.142} \\
\hline Grade 3 & 67 & $36.7 \pm 2.7$ & & $33.0 \pm 3.1$ & \\
\hline $\mathrm{Ml}<50 \%$ & 51 & $42.4 \pm 1.9$ & \multirow{2}{*}{0.163} & $41.0 \pm 2.3$ & \multirow{2}{*}{0.106} \\
\hline $\mathrm{Ml} \geq 50 \%$ & 69 & $36.2 \pm 3.0$ & & $32.7 \pm 3.3$ & \\
\hline $\mathrm{Cl}(-)$ & 91 & $42.6 \pm 1.5$ & \multirow{2}{*}{$<0.001^{*}$} & $41.6 \pm 1.8$ & \multirow{2}{*}{$<0.001^{*}$} \\
\hline $\mathrm{Cl}(+)$ & 29 & $25.8 \pm 4.8$ & & $17.8 \pm 4.6$ & \\
\hline LNM (-) & 107 & $41.0 \pm 1.7$ & \multirow{2}{*}{$0.001^{*}$} & $39.3 \pm 1.9$ & \multirow{2}{*}{$<0.001^{*}$} \\
\hline LNM (+) & 13 & $17.8 \pm 4.8$ & & $9.1 \pm 3.7$ & \\
\hline DM (-) & 103 & $40.9 \pm 1.7$ & \multirow{2}{*}{$<0.001^{*}$} & $39.2 \pm 2.0$ & \multirow{2}{*}{$<0.001^{*}$} \\
\hline $\mathrm{DM}(+)$ & 17 & $17.4 \pm 3.7$ & & $8.0 \pm 2.2$ & \\
\hline
\end{tabular}

OS — overall survival; PFS — progression free survival; LVI — lymphovascular invasion; $\mathrm{MI}$ - myometrial invasion; $\mathrm{Cl}$ — cervical invasion; LNM — lymph node metastasis; DM - distant metastasis; significant values $(P<0.05)$ are indicated with *

\section{DISCUSSION}

Most patients with EC are diagnosed in the early stage (FIGO 1-2) [6]. Five-year survival rates are $74-91 \%$ in early stage disease, $57-66 \%$ in stage 3 and $20-26 \%$ in stage 4 [6]. Aside from the disease stage, tumor histology, grade, depth of $\mathrm{MI}, \mathrm{LVI}$, presence of LNM and the patient's age can be counted among the clinicopathological prognostic factors [15]. Tumor size and various molecular factors have also been suggested to be of prognostic value in studies, although this is still under research [15]. However, survival may be different even in patients with the same risk factors. Furthermore, a large proportion of the known risk factors rely on staging during extensive surgery, which may be unnecessary in some patients with early-stage disease, and this is the subject of ongoing researches aimed at identifying preoperative noninvasive prognostic factors. FDG PET/CT is an effective imaging method that is used for initial diagnosis, staging, re-staging and evaluating response to therapy in a wide variety of cancers [16]. In the initial staging of endometrial cancer, $\mathrm{PET} / \mathrm{CT}$ is recommended to detect extrauterine disease [17]. In addition, PET/CT has been shown to be useful in identifying candidates for surgical staging [18] and in determining post-operative recurrence [19]. But, only a limited number of studies evaluated the prognostic value of $\mathrm{PET} / \mathrm{CT}$ in EC, and the results to date have been conflicting. In a study of 100 patients with stage 1-4EC, Walentowicz-Sa- dlecka et al. [8] identified significantly shorter OS in patients with a preoperative SUVmax $\geq 17.7$ than in patients with a preoperative SUVmax of $<17.7$. Another study involving 42 patients with EC (stage $3 C-4$ ) found significantly longer OS in patients with a low SUVmax of the primary tumor $(<9.5)$ or lymph node $(<7.3)$ than in patients with a higher SUVmax [5]. In a study of 84 patients with stage 1-4 EC by Shim et al. [7], preoperative MTV and TLG were identified as independent prognostic factors for PFS in EC, although no significant relationship was identified between PFS and SUVmax and SUVmean, or between OS and MTV and TLG values. In a study by Liu et al. [20] involving 15 patients with stage 4B EC, whole body MTV and whole body TLG were found to be significant prognostic factors for survival. The present study found no significant relationship between the SUVmax, SUVmean, MTV and TLG values of the primary tumor and OS and PFS. The heterogeneity of the study population in terms of such clinicopathological factors as stage, histology and grade is believed to be the cause of variety in the results. Furthermore, whole body measurements were carried out in the study by Liu et al., whereas the present study evaluated the PET parameters of the primary tumor. Such methodological differences may have also caused the differences in the results.

The present study identified FIGO stage, Cl, LVI, and the presence of LNM and DM as prognostic factors for OS 
and PFS, while age, tumor diameter, histology, grade, and MI were not found to be related to OS and PFS. Various risk classification systems have been developed to predict prognosis and to guide treatment of endometrial cancer. Risk factors such as FIGO stage, tumor histology, grade, $\mathrm{MI}$ and LVI are evaluated together in these classification systems [15]. However, in our study, we discussed the risk factors one by one. And so, the non-homogeneous distribution of other prognostic factors across the age groups and patients classified in terms of tumor histology, grade, diameter and MI degree, may have also resulted in the difference in the current findings. For example, LNM was mostly present in patients aged $<62$ years. In a meta-analysis including 14 studies (672 patients), complete cytoreduction to no gross residual disease for patients with advanced or recurrent endometrial cancer was found to be associated with superior overall survival outcome [21]. However, we did not evaluate the presence of post-treatment residual tumor. Furthermore, although all patients in the present study underwent surgery, post-operative treatments were not standard. Differences in the treatment management may have contributed to current results. In addition, some micrometastases may not be detected during surgery or by PET/CT. The presence of undetectable micrometastasis may also have affected prognosis. AlHilli et al. [22] declared a relationship between tumor diameter and survival. In that study, $2 \mathrm{~cm}$ was utilized for the cut-off value of the tumor size [22]. But we used a threshold of $4 \mathrm{~cm}$ that may have caused us to find a different result.

In the present study, 5-year survival rates were not evaluated and there were limitations such as relatively short follow-up period and retrospective design.

\section{CONCLUCIONS}

No significant relationship was found between PET parameters and OS and PFS, and so prospective studies involving a larger number of patients with more homogeneous groups are needed.

\section{REFERENCES}

1. Kitajima K, Kita M, Suzuki K, et al. Prognostic significance of SUVmax (maximum standardized uptake value) measured byFDG PET/CT in endometrial cancer. Eur J Nucl Med Mol Imaging. 2012; 39(5): 840-845, doi: 10.1007/s00259-011-2057-9, indexed in Pubmed: 22349717.

2. Kwon JS. Improving survival after endometrial cancer: the big picture. J Gynecol Oncol. 2015; 26(3): 227-231, doi: 10.3802/jgo.2015.26.3.227, indexed in Pubmed: 26197859.

3. Lewin SN, Herzog TJ, Barrena Medel NI, et al. Comparative performance of the 2009 international Federation of gynecology and obstetrics' staging system for uterine corpus cancer. Obstet Gynecol. 2010; 116(5): 1141-1149, doi: 10.1097/AOG.0b013e3181f39849, indexed in Pubmed: 20966700.

4. Braun MM, Overbeek-Wager EA, Grumbo RJ. Diagnosis and Management of Endometrial Cancer. Am Fam Physician. 2016; 93(6): 468-474, indexed in Pubmed: 26977831.

5. Kim HJ, Choi J, Jeong YH, et al. Prognostic Value of Metabolic Activity Measured by (18)F-FDG PET/CT in Patients with Advanced Endo- metrial Cancer. Nucl Med Mol Imaging. 2013; 47(4): 257-262, doi: 10.1007/s13139-013-0228-2, indexed in Pubmed: 24900121.

6. Creasman WT, Odicino F, Maisonneuve $P$, et al. Carcinoma of the corpus uteri. FIGO 26th Annual Report on the Results of Treatment in Gynecological Cancer. Int J Gynaecol Obstet. 2006; 95 Suppl 1: S105-S143, doi: 10.1016/S0020-7292(06)60031-3, indexed in Pubmed: 17161155.

7. Shim SH, Kim DY, Lee DY, et al. Metabolic tumour volume and total lesion glycolysis, measured using preoperative 18F-FDG PET/CT, predict the recurrence of endometrial cancer. BJOG. 2014; 121(9): 1097-1106; discussion 1106, doi: 10.1111/1471-0528.12543, indexed in Pubmed: 24397772.

8. Walentowicz-Sadlecka M, Malkowski B, Walentowicz P, et al. The preoperative maximum standardized uptake value measured by $18 \mathrm{~F}-\mathrm{FDG}$ $\mathrm{PET} / \mathrm{CT}$ as an independent prognostic factor of overall survival in endometrial cancer patients. Biomed Res Int. 2014; 2014: 234813, doi: 10.1155/2014/234813, indexed in Pubmed: 24719847.

9. Berghmans $T$, Dusart M, Paesmans $M$, et al. European Lung Cancer Working Party for the IASLC Lung Cancer Staging Project. Primary tumor standardized uptake value (SUVmax) measured on fluorodeoxyglucose positron emission tomography (FDG-PET) is of prognostic value for survival in non-small cell lung cancer (NSCLC): a systematic review and meta-analysis (MA) by the European Lung Cancer Working Party for the IASLC Lung Cancer Staging Project. J Thorac Oncol. 2008; 3(1): 6-12, doi: 10.1097/JTO.0b013e31815e6d6b, indexed in Pubmed: 18166834.

10. Pan L, Gu P, Huang G, et al. Prognostic significance of SUV on $\mathrm{PET} / \mathrm{CT}$ in patients with esophageal cancer: a systematic review and meta-analysis. Eur J Gastroenterol Hepatol. 2009; 21(9): 1008-1015, doi: 10.1097/MEG.0b013e328323d6fa, indexed in Pubmed: 19352191.

11. Casasnovas RO, Meignan M, Berriolo-Riedinger A, et al. Groupe d'étude des lymphomes de l'adulte (GELA). SUVmax reduction improves early prognosis value of interim positron emission tomography scans in diffuse large B-cell lymphoma. Blood. 2011; 118(1): 37-43, doi: 10.1182/blood-2010-12-327767, indexed in Pubmed: 21518924.

12. Itti E, Lin C, Dupuis J, et al. Prognostic value of interim 18F-FDG PET in patients with diffuse large B-Cell lymphoma: SUV-based assessment at 4 cycles of chemotherapy. J Nucl Med. 2009; 50(4): 527-533, doi: 10.2967/jnumed.108.057703, indexed in Pubmed: 19289424.

13. Scott $A M$, Gunawardana $D H$, Wong J, et al. Positron emission tomography changes management, improves prognostic stratification and is superior to gallium scintigraphy in patients with low-grade lymphoma: results of a multicentre prospective study. Eur J Nucl Med Mol Imaging. 2009; 36(3): 347-353, doi: 10.1007/s00259-008-0958-z, indexed in Pubmed: 18931840.

14. Boellaard R, O'Doherty MJ, Weber WA, et al. FDG PET and PET/CT: EANM procedure guidelines for tumour PET imaging: version 1.0. Eur J Nucl Med Mol Imaging. 2010; 37(1): 181-200, doi: 10.1007/s00259-009-12974, indexed in Pubmed: 19915839.

15. Colombo N, Creutzberg C, Amant F, et al. ESMO-ESGO-ESTRO Endometrial Consensus Conference Working Group. ESMO-ESGO-ESTRO Consensus Conference on Endometrial Cancer: diagnosis, treatment and follow-up. Ann Oncol. 2016; 27(1): 16-41, doi: 10.1093/annonc/mdv484, indexed in Pubmed: 26634381.

16. Maldonado A, González-Alenda FJ, Alonso M, et al.PET-CT in clinical oncology. Clin TransI Oncol. 2007; 9(8):494-505, indexed in Pubmed: 17720652.

17. NCCN Practice Guidelines Narrative Summary of Indications for FDG PET and PET/ CT. 2/14/2016 ed2016.

18. Özgü E, Öz M, YıldızY, et al. Prognostic value of 18F-FDG PET/CT for identifying high- and low-risk endometrial cancer patients. Ginekol Pol. 2016; 87(7): 493-497, doi: 10.5603/GP.2016.0032, indexed in Pubmed: 27504941.

19. Bollineni VR, Ytre-Hauge S, Bollineni-Balabay O, et al. High Diagnostic Value of 18F-FDG PET/CT in Endometrial Cancer: Systematic Review and Meta-Analysis of the Literature. J Nucl Med. 2016; 57(6): 879-885, doi: 10.2967/jnumed.115.170597, indexed in Pubmed: 26823564.

20. Liu FY, Chao A, Lai CH, et al. Metabolic tumor volume by $18 \mathrm{~F}-\mathrm{FDG}$ PET/CT is prognostic for stage IVB endometrial carcinoma. Gynecol Oncol. 2012; 125(3): 566-571, doi: 10.1016/j.ygyno.2012.03.021, indexed in Pubmed: 22440787.

21. Barlin JN, Puri I, Bristow RE. Cytoreductive surgery for advanced or recurrent endometrial cancer: a meta-analysis. Gynecol Oncol. 2010; 118(1): 14-18, doi: 10.1016/j.ygyno.2010.04.005, indexed in Pubmed: 20434198.

22. AlHilli MM, Mariani A, Bakkum-Gamez JN, et al. Risk-scoring models for individualized prediction of overall survival in low-grade and high-grade endometrial cancer. Gynecol Oncol. 2014; 133(3): 485-493, doi: 10.1016/j.ygyno.2014.03.567, indexed in Pubmed: 24690476. 